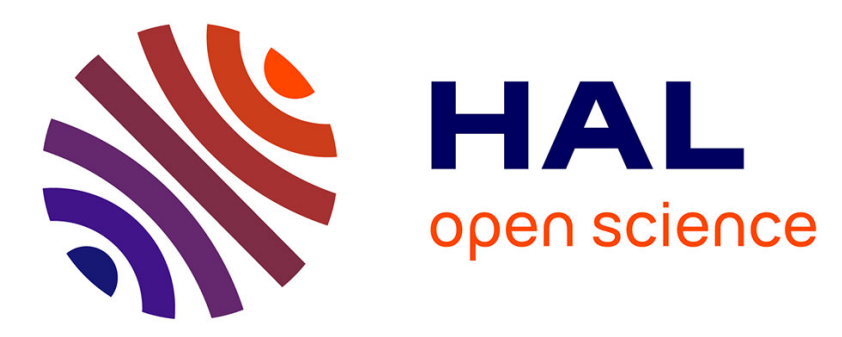

\title{
Functional brain neuroimaging-guided repetitive transcranial magnetic stimulation in neurodevelopmental disorders: The case of a schizencephaly-related spastic dystonia
}

Ksenija Vucurovic, Samuel Emeriau, Jean-Michel Coulon, Philippe Peruzzi, Claude-Fabien Litré, Arthur Kaladjian

\section{To cite this version:}

Ksenija Vucurovic, Samuel Emeriau, Jean-Michel Coulon, Philippe Peruzzi, Claude-Fabien Litré, et al.. Functional brain neuroimaging-guided repetitive transcranial magnetic stimulation in neurodevelopmental disorders: The case of a schizencephaly-related spastic dystonia. Journal of the Neurological Sciences, 2017, 10.1016/j.jns.2017.05.012 . hal-01522231

\section{HAL Id: hal-01522231 \\ https://hal.science/hal-01522231}

Submitted on 13 May 2017

HAL is a multi-disciplinary open access archive for the deposit and dissemination of scientific research documents, whether they are published or not. The documents may come from teaching and research institutions in France or abroad, or from public or private research centers.
L'archive ouverte pluridisciplinaire HAL, est destinée au dépôt et à la diffusion de documents scientifiques de niveau recherche, publiés ou non, émanant des établissements d'enseignement et de recherche français ou étrangers, des laboratoires publics ou privés. 


\section{Letter to the Editor}

\section{Functional brain neuroimaging-guided repetitive transcranial magnetic stimulation in neurodevelopmental disorders: The case of a schizencephaly-related spastic dystonia}

\section{Keywords:}

Schizencephaly

rTMS

Brain plasticity

Brain development

Spastic dystonia

\section{Dear Editor,}

Spastic dystonia is defined as tonic involuntary muscle activation at rest superimposed over spastic paresis [1]. It occurs in different pathological conditions, ranging from dopamine-dependent dystonia [2] to post-stroke deforming spastic hemiparesis [1]. Even though important burden is associated to spastic dystonia, therapeutic options are scarce and mostly limited to intramuscular botulinum toxin injection and surgical partial nerve section. Repetitive transcranial magnetic stimulation (rTMS) has been proposed as an interesting therapeutic option, but with inconsistent results [3]. We suggest that optimized targeting based on functional brain imaging could enhance the results of rTMS in schizencephaly-related dystonia and improve our knowledge about the technical procedure to become more widely applicable in neurodevelopmental disorders.

Here we report the case of 41 years old woman suffering from openlip schizencephaly-related left hemiparesis with spastic dystonia. Schizencephaly, a disorder of cortical development, consists of a gray matter polymicrogyri-lined cleft of one cerebral hemisphere connecting extra-axial subarachnoid space and ventricle [4]. The patient was born after eight months pregnancy without reported complication. During childhood, congenital limb length discrepancy and equinus foot deformity were surgically corrected. A left spastic hemiparesis appeared in early childhood. It was associated to abnormal synkinesia that referred to involuntary movement of right hand and to a lesser extent of left foot that occurred with voluntary movement of left hand and vice versa (Supplementary Video 1). Neither spastic paresis nor other movement disorder was found in the right-side extremities. A painful dystonic gait pattern began on both left-side extremities around forty years of age and severely compromised the patient's autonomy. Its management by intramuscular botulinum toxin injections was painful and only partially effective, so her physician referred her for rTMS treatment trial. Based on a literature review, we hypothesized that reducing the excitability of primary motor cortex involved in motor control of left-side extremities would be effective in spastic dystonia treatment.

First, we measured the motor evoked potentials (MEP) for left abductor pollicis brevis (APB) muscle in a relaxed state in order to determine motor threshold (MT). Single-pulse TMS was applied with a biphasic waveform using a figure-of-eight coil connected to a MagPro R30 MagOption stimulator (MagVenture, Denmark). The coil was positioned tangentially with the handle rotated posterior-laterally $45^{\circ}$ to the sagittal plane to induce a posterior-anterior current flow in the brain. Motor responses were recorded using MEP Monitor (MagVenture, Denmark) and pre-gelled surface electrodes. It is noteworthy that no MEP was evoked in left APB when the motor cortex of the right hemisphere facing the schizencephaly cleft was stimulated, even when stimulation intensity was increased to $100 \%$ of maximal stimulator output (MSO). Conversely, MEPs in both left and right APB were simultaneously evoked at 3 different sites of left motor cortex stimulation. The MT, defined as minimal intensity that induced $50 \mu \mathrm{V}$ MEP of APB in at least five out of ten stimulations, was found over C3 (according to the 10-20 International System for EEG electrodes). It was of $45 \% \mathrm{MSO}$ for right APB and 50\%MSO for the left one. The MEPs of both muscles evoked by stimulation over C3 at 120\% of MT showed similar latencies (left: $23.6 \mathrm{~ms}$, right: $24.1 \mathrm{~ms}$; mean of 5 consecutives measures), amplitudes (left: $1320 \mu \mathrm{V}$, right: $1260 \mu \mathrm{V}$; mean of 5 consecutives measures) and figures of potential (Fig. 1A). As previously reported in other schizencephaly cases $[4,5]$, these results suggest that the corticomotoneuronal tract to both hands originates from the non-affected motor cortex.

Secondly, in order to precisely target rTMS, we performed functional magnetic resonance imaging (fMRI) with a block-designed motor paradigm to show sensorimotor regions involved in left hand and foot movements. The patient was instructed to alternate between left hand finger to thumb movements during activation periods (30 s) and resting-state periods ( $30 \mathrm{~s}$ ), for a total imaging time of $6 \mathrm{~min}$. Synkinetic movements of the right hand and left foot were noted during voluntary movements of the left hand. Anatomical MRI showed right centrally located open-lip schizencephaly (Fig. 1B), and fMRI revealed a dramatically rearranged brain map with activation of left precentral gyrus during movements of the left hand (Fig. 1C).

Finally, we applied low-frequency rTMS over the fMRI-identified left primary motor cortex for targeting the left hand and foot representation. The same setup was used for single-pulse TMS and rTMS. Stimulation was performed as continuous $1 \mathrm{~Hz}$ rTMS train of 1200 pulses at $100 \%$ of resting MT. Both targets were localized by fMRI-guided neuronavigation. This rTMS procedure was performed once a day for three consecutive days.

Unified Dystonia Rating Scale (UDRS) was used to evaluate dystonia severity. Briefly, severity and duration of dystonia based on selfreported patient's evaluation and clinical assessment are rated in fourteen body areas on a scale from 0 to 4 for both factors. The total score of the UDRS is the addition of severity and duration factors for all affected body areas. Thus, the maximal total score of UDRS is 112 . Clinical assessment identified four affected areas in the patient: left shoulder and proximal arm, left distal arm and hand including elbow, left pelvis and proximal leg, left distal leg 

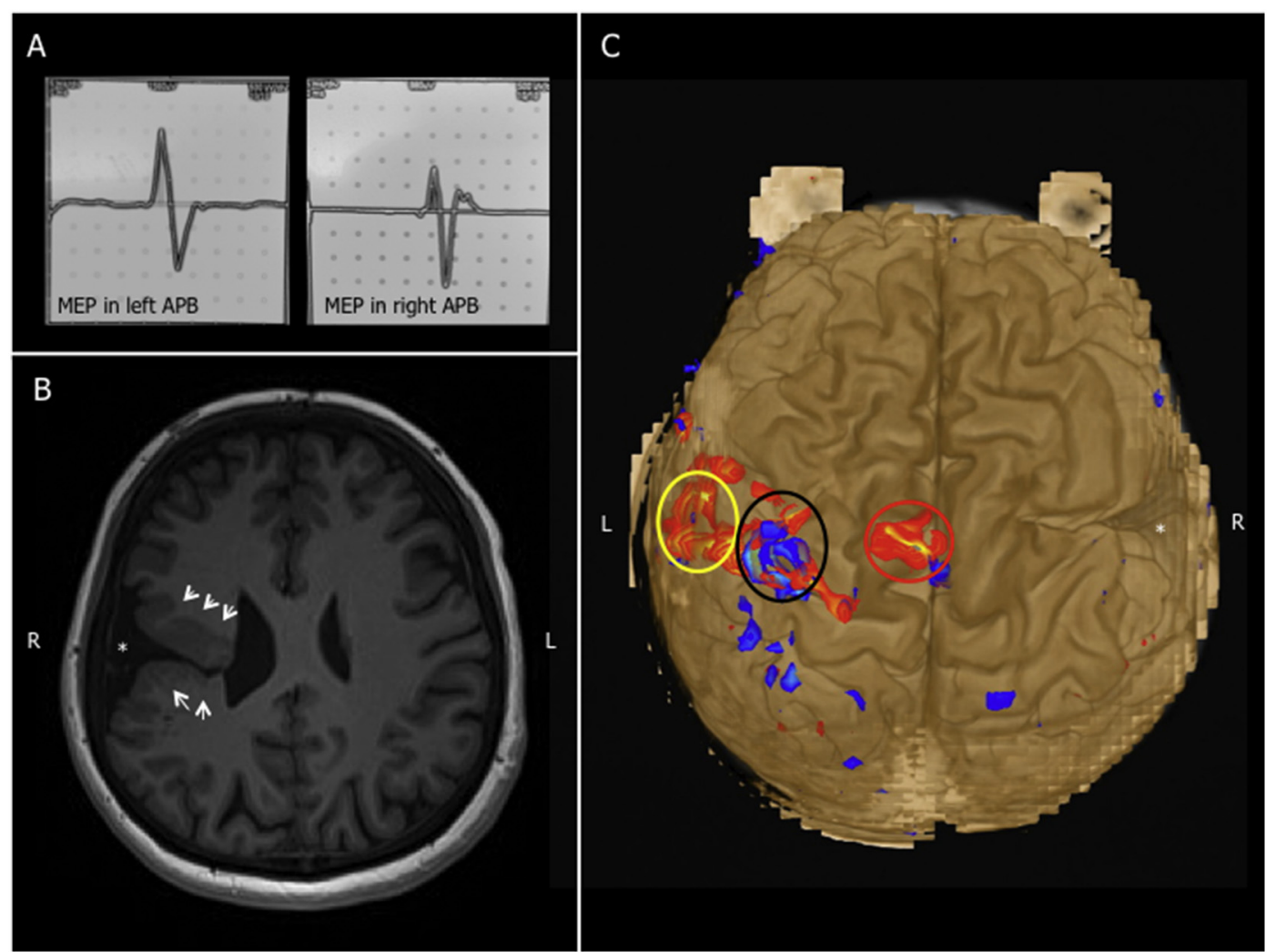

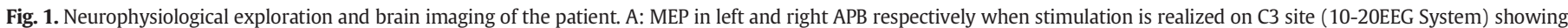

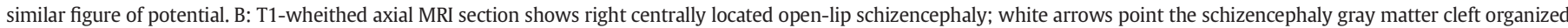

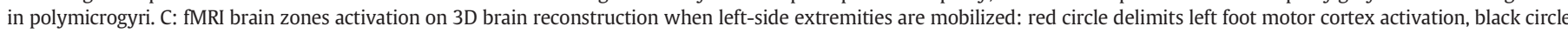

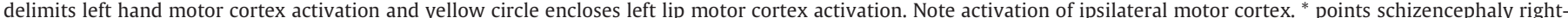

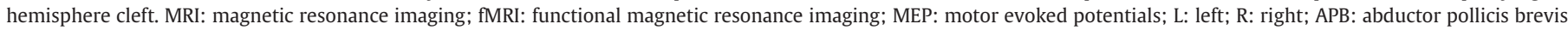
muscle. (For interpretation of the references to colour in this figure legend, the reader is referred to the web version of this article.)

and foot including knee. The UDRS score of 32 before treatment decreased to 22 after three days long procedure and reached 24 one month later (Supplementary Table 1). In addition, patient reported better movement flexibility and improved autonomy (Supplementary Video 2). No side effects were reported.

Our results demonstrate that the left precentral gyrus is the only functional primary motor cortex for both sides of patient's body. It was suggested that ipsilateral cortico-spinal fibers for motor control are present during perinatal period, but they become rapidly non-functional during typical brain development because the contralateral motor pathway is enhanced [4]. In schizencephaly, a prenatal cortical lesion probably prevents formation of a typical contralateral pathway and thus ipsilateral cortico-spinal motor connections are strengthened in postnatal period.

We used fMRI to investigate reorganization of sensorimotor cortex and cortico-spinal tract in response to schizencephaly in order to optimize a therapeutic rTMS procedure. This case report highlights the importance of targeting functional rather than anatomical brain zone in neuromodulation treatment, particularly in neurodevelopmental disorders.

Supplementary data to this article can be found online at http://dx. doi.org/10.1016/j.jns.2017.05.012.

\section{Conflicts of interest}

None of the authors have potential conflicts of interest to be disclosed.
The article has not been and will not be published elsewhere in the same form.

The submitting author has circulated the article and secured the final approval of the version to be peer-reviewed from all co-authors prior to the publication of the article.

\section{Funding}

Sponsors were not involved in this study.

\section{Acknowledgement}

We would like to thank our patient, M-C.L., for her participation in this study and her consent for publication of clinical data.

\section{References}

[1] J.M. Gracies, Coefficients of impairment in deforming spastic paresis, Ann. Phys. Rehabil Med. 58 (3) (Jun 2015) 173-178, http://dx.doi.org/10.1016/j.rehab.2015.04.004.

[2] S. Wijemanne, J. Jankovic, Dopa-responsive dystonia-clinical and genetic heterogeneity, Nat. Rev. Neurol. 11 (7) (Jul 2015) 414-424, http://dx.doi.org/10.1038/ nrneurol.2015.86 (Review)

[3] J.P. Lefaucheur, N. André-Obadia, A. Antal, S.S. Ayache, C. Baeken, D.H. Benninger, et al., Evidence-based guidelines on the therapeutic use of repetitive transcranial magnetic stimulation (rTMS), Clin. Neurophysiol. 125 (11) (Nov 2014) 2150-2206 http://dx.doi.org/10.1016/j.clinph.2014.05.021 (Review).

[4] Y. Vandermeeren, A. De Volder, E. Bastings, J.L. Thonnard, J. Duque, C. Grandin, et al., Functional relevance of abnormal fMRI activation pattern after unilateral schizencephaly, Neuroreport 13 (14) (Oct 7 2002) 1821-1824. 
[5] P. Belardinelli, L. Ciancetta, M. Staudt, V. Pizzella, A. Londei, N. Birbaumer, et al., Cerebro-muscular and cerebro-cerebral coherence in patients with pre- and perinatally acquires unilateral brain lesions, NeuroImage 37 (4) (Oct 1 2007) 1301-1314.

Ksenija Vucurovic C2S Laboratory (EA 6291), University of Reims Champagne-Ardenne, Reims, France

Department of Neurosurgery, Maison Blanche Hospital, Reims University Hospital, Reims, France

Corresponding author at: Laboratoire C2S (EA 6291), UFR Lettres et Sciences humaines, 57, rue Pierre Taittinger, 51096 Reims Cedex,

France.

E-mail address: vksenija@yahoo.com.

Samuel Emeriau

Department of Medical Imaging, Maison Blanche Hospital, Reims University Hospital, Reims, France
Jean-Michel Coulon Department of Physical Medicine and Rehabilitation, Sébastopol Hospital, Reims University Hospital, Reims, France

Philippe Peruzzi Claude-Fabien Litré Department of Neurosurgery, Maison Blanche Hospital, Reims University Hospital, Reims, France

Arthur Kaladjian C2S Laboratory (EA 6291), University of Reims Champagne-Ardenne, Reims, France Department of Adult Psychiatry, Robert Debré Hospital, Reims University Hospital, Reims, France

9 February 2017 Available online 10 May 2017 